\title{
An artificial immune network based novel approach to predict short term load forecasting
}

\author{
Arpita Samanta Santra ${ }^{1 *}$, Cheng-Chin Taso ${ }^{2}$, Pei-Chann Chang ${ }^{3}$
}

\author{
1, 2, 3 Department of Information Management, Yuan Ze University, Chung-Li, Taiwan
}

\author{
Index Terms \\ Short-term Load Forecasting \\ Immune Memory \\ Immune Networks
}

Received: 12 December 2016

Accepted: 3 May 2017

Published: 30 June 2017

\begin{abstract}
Recent trends of Short-Term Load Forecasting (STLF) is a key issue to regulate power in the electricity market. Many researchers have performed research in this area but it still needs an accurate and robust load forecast method. In this paper, we propose a novel Artificial Immune Network (AIN)-based approach to predict forecast load depending on last three days' mean actual load. The approach creates an immune memory using time series to forecast one-day ahead hourly loads. The method takes hourly loads separately as an individual daily time series and considers it as an antigen, an affinity is calculated between an antigen and antibody in Immune Networks (INs). A cross-reactivity threshold is used to find the appropriate cluster for an antigen in an immune network. The historical dataset of Poland is trained and tested by this method which predicts more accurately compared to the most recently existing STLF methods, such as simple Nearest Neighbor (NN), Multilayer Perceptron (MLP), Fuzzy Estimators (FE), and Artificial Immune System (AIS).
\end{abstract}

\section{INTRODUCTION}

Electricity load forecasting is a relation between production and energy consumption; it is an inbuilt method of planning, design, and operation of electrical components. Depending on time duration, load forecasting methods are classified into three types: Long-Term Load Forecasting (LTLF) predicts more than one-year forecasting loads, Medium-Term Load Forecasting (MTLF) predicts forecasting loads between one week and one year, and short-term load forecasting predicts forecasting load between one hour and one week. STLF is a crucial time series prediction technique, which has been used to perform the forecasting task in power system to control the energy management system and to increase operational efficiency. Accurate prediction of load forecasting is essential to optimize unit commitment, economic dispatch, hydro scheduling, hydro co-ordination, spinning reverse allocation, and interchange evaluation. An accurate predicted load can save a lot of money and electricity, e.g. an increase of only $1 \%$ in forecast error caused an increase of 10 million pounds in operating cost per year for one electric utility in the United Kingdom [1].

Electricity market cannot function effectively without predicting forecast load. At every instance, electricity load is balanced by an operation of power system. In electricity companies, load forecasting is managed in a secure and efficient way of production, transmission, and distribution of electricity. Mostly mentioned, there are two concerns; one is prediction model selection and other is data selection that should be treated in STLF. Forecast is defined by prediction of future events and situations. Forecast model can be classified into conventional and unconventional model. Conventional STLF system applies statistical analysis, regression method [2], and smoothing techniques. In the last few decades, many several newer forecasting approaches have been used like stochastic process, Autoregressive Moving Average (ARMA) models [3], data mining models, and most popularly used Artificial Intelligence (AI),

* Corresponding author: Arpita Samanta Santra

†Email: santraarpita83@gmail.com 
such as Artificial Neural Networks (ANN), Fuzzy Inference Systems (FIS), neuro-fuzzy systems [4], Support Vector Machines (SVM), and AIS [5] that have been achieving good performance and tackling uncertainties in load forecasting application.

Neural Network (NN) is a series of algorithms that attempts to identify underlying relationships in a set of data. It has the ability to adapt and to change input for producing the best possible result without redesigning the output criteria. The useful features like universal approximation property, learning ability, and robustness to noise in data can be used in STLF technique, but it is difficult to find an optimal architecture, weak extrapolation capacity, and many parameters' estimates.

The problem solving control system fuzzy logic allows imprecise, incomplete, and ambiguous information to be mentioned in the STLF model. The method can be effectively used with uncertainty. In the learning process of neuro-fuzzy systems, the knowledge for building IF-THEN rule base is obtained directly from data in the learning phase. The structure of neuro-fuzzy system is complex and parameters are large. Therefore, it does not guarantee convergence to the global minimum.

AIN has also been employed in STLF to find the best performance in dealing with time series without any complex mathematical formulations. These systems solve many computational problems from mathematics, engineering and information technologies. The advantages of AIN are its learning and memorization intelligence. Anyone can measure the average prediction forecast error to predict perfection of load forecast to compare with the actual load of the same day.

Proposed approach can be summarized as follows:

- A novel AIN-based clustering approach is presented here for solving STLF problems. To the best of our knowledge, this STLF method has never been presented in the literature.

- The method has two phases: training phase and testing phase; in training phase, the model is trained to produce INs. Each IN has more than one cluster and each cluster has an antibody. A cluster is created by adjustable crossreactivity threshold $(\theta)$ and the antibody is recalculated by means of inserted antigen and cluster's antibody. In testing phase, an antigen is generated from last three days' mean actual load. A cluster is selected from an IN based on $\theta$ and the antibody of that cluster is the forecasting load for that antigen.

- Forecasted errors are calculated between actual load and predicted load. However, this paper focuses on the hourly load forecasting, which is the most effective form of STLF, though the proposed method can be easily extended to daily peak load prediction.

The rest of the paper is organized as follows: literature survey of related works is described in Section II; Section III describes the proposed AIN method; experimental results of proposed work and comparison with related works are examined in Section IV; Section V describes discussion for our method; finally, conclusion and future work of the proposed method are described in Section VI.

\section{LITERATURE REVIEW}

Load forecasting technique has been started from mid-1960s [6], [7]. The accuracy of load forecast has a vital effect on electricity system and can change the economic growth of any country. In last few decades, a lot of research has been done to tune load forecast accuracy. Some of the STLF methods to predict system loads are discussed here. Autoregressive Integrated Moving Average (ARIMA) [8], a linear prediction technique that was a generalization of ARMA [9], was used for time series load forecasting. This method was cascading into two methods: non-stationary and wide-sense stationary. ARIMA models [10] are relatively robust, efficient, and more complex structural models in relation to short-term forecasting. Another approach to predict the system load in STLF is fuzzy logic system, which is a well-known rule-based approach. It can recognize and evaluate any unknown data on the compact set to arbitrary accuracy from input data. Fuzzy logic is a technique to map input data to output data. In article [11], authors find rules to map similarities from huge data. Fuzzy logic system is very robust in load forecasting system. After mapping fuzzy inputs and set of rules, a centroid defuzzification is used to identify such precise outputs [12], [13], [14].

A nonlinear prediction model, ANN is widely used in prediction technique for its learning capability. There are several types of ANN such as feedforward, self-organizing, etc. In book [15], authors proposed MLP model, which predicted system loads in STLF. The approach had multilayer nodes in a directed graph. Data processing occurs in every layer except the input layers. Each node had a nonlinear activation function. The model was trained by backpropagation technique. A fully connected three-layer feedforward ANN-based approach was proposed in [16] to estimate the load in STLF and applied a backpropagation method to update the weights, in an attempt to minimize the loss function. In this article, authors considered temperature as a 
climatic variable and could predict system load from one day to seven days. Many research works have been done in last few decades using ANN in combination with other techniques like regression tree model [17], fuzzy logic system [14], and time series method [18].

\section{A. Artificial Immune System}

A Natural Immune System (NIS) is one of the most complex with several functional biological systems to identify the harmful substances (e.g. bacteria and viruses) by molecules called antigens. An immune response consists of secretion of antibody to participate in the recognition and destruction that is triggered by antigens. AIS algorithm is adapted from Biological Immune System (BIS). AIS is a type of computational intelligence system taken from the memorized immune system. This is an attractive and emerging field involving models, techniques, and applications of great diversity [19]. AIS development can be classified into two target fields. (i) Engineering problem solving by adapting Immune system concept. (ii) The provision and simulation of models for immune system theories. The basic idea of this Evolutionary Algorithm (EA) is simulated by adopting selection, crossover, mutation, and replacement operators [20].

In [21], authors developed BIS to protect them from various foreign attacks such as virus, bacteria, and other parasites, which are treated as antigen. Immune system can recognize self or non -self cell in a body where non-self cell is also known as antigen (Ag). B-cells are an essential component of humoral immune response to generate antibody (Ab) recognition and tie up with Ags [22]. The features of EA like robustness and ability to adapt in changing environment is the key secret behind switching from traditional learning approach to EA [23]. In [24], authors proposed an Improved Bacterial Chemotaxis Optimization (IBCO); the method was added in Back Propagation Neural Network (BPN) to generate an effective forecasting model for prediction of various stock indicates. The method had less computational complexity, better prediction accuracy, and less training time compared to BPN model [25]. An immune response-based immunological algorithm using clonal selection technique of AIS is used for numerical and combinatorial optimization. A clonal selection algorithm, CLONALG [26], [27] takes a set of antigens and produces a set of memory to recognize unseen pattern. AIS can be used together with other soft computing techniques to create greater approach and to find out a better solution.
A B-cell is selected based on their affinity maturation. A higher affinity clone has replaced the lower affinity cell of early generation. An Artificial Immune Recognition System (AIRS) [28] is reinforcement-learning approach based on clonal selection method that is used to classify unseen item. AIRS algorithm acts as a pre-computation to the k- nearest neighbor approach, which works well on certain type of classification problem [29].

\section{B. Artificial Immune Network}

Immune network theory [30] was invented by Jerne in 1974, where immune system maintains an idiotypic network of interconnected cells for antigen recognition. These cells both stimulate and suppress each other in a certain way that leads to stablizing the networks. The formation of such a network was possible by the presence of paratope and idiotope on each antibody cell. The paratope present on one B-cell was recognized by other B-cells idiotopes. Immune network algorithms have been used in clustering, data visualization, control, optimization domains, and share properties with artificial neural networks [31]. AIN has also been used in collaborating filtering [32], [33], [34] and patent quality classification model [35].

\section{PROPOSED METHOD}

STLF problem is solved by proposed method, which takes historical dataset of $m$ days as input data. $75 \%$ of historical data is treated as training dataset and rest $25 \%$ is considered as testing dataset. The method generates system load for each hour of next day. Forecast errors are the distinction between actual load and forecast load generated by proposed model. In training phase, the model creates 24 immune networks of vector $\mathrm{n}$ whose components are the following hourly loads. It is considered that information about future prediction of the load time series is included in forecast model. This assumption for load time series is characterized as annually, half-yearly, quarterly, monthly, and daily cycle due to changes in industrial activities, and climate conditions are confirmed by statistical tests.

Let $\mathrm{nx}$ be a vector of xth hour power system loads of each day in training dataset. $\mathrm{n}_{x}=\left[\mathrm{n}_{x}(1)\right.$, $\left.\mathrm{n}_{x}(2), \ldots, \mathrm{n}_{x}(3 \mathrm{~m} / 4)\right]$, where $\mathrm{n}_{x}$ (1) represents the xth hour system load of day one in training dataset and assume ny be a vector of hourly loads of the day of forecast and $\mathrm{n}_{y}=$ $\left[\mathrm{n}_{y}(1), \mathrm{n}_{y}(2), \ldots, \mathrm{n}_{y}(24)\right]$ represents each hour system load of predicted day. Proposed method learns to map $x \rightarrow y$. After learning input pattern, $x$ is presented to the method and pat- 
tern y is obtained as a method output. The method is based on AIN, where concatenate patterns $\mathrm{x}$ and $\mathrm{y}$ form antigens. Each antigen is one-hour system load of a day, which is actual load of the previous day. The task of immune system is to learn to map the set of antigens to the set of antibodies.

The immune memory is an effect of learning method where a network is created based on hourly system load of each day of training data and these loads are treated as antigens of the network. A group of clusters is created for each network and every cluster has an antibody. An affinity value is calculated between an antigen and an antibody of each cluster. If the affinity value is less than or equal to the crossreactivity threshold value $(\theta)$, then antigen is placed in that cluster and the antibody is reformulated by means of the previous antibody of cluster and the antigen. If no cluster is selected for that antigen, a new cluster is created and it is set as antibody of the newly created cluster.

In testing phase, a new antigen of each hour for forecasting day is created by calculating the mean value of three consecutive previous days' actual loads of the same hour. This new antigen is proceeded to the same hour immune network and the cluster for which affinity value is minimum is selected. The quality creation of the proposed method is the forecasted error. The forecast error is calculated by Mean Absolute Percent Error (MAPE), which is calculated between predicted load and actual load of the same hour of the predicted day. The framework of proposed method is shown in Figure 1.

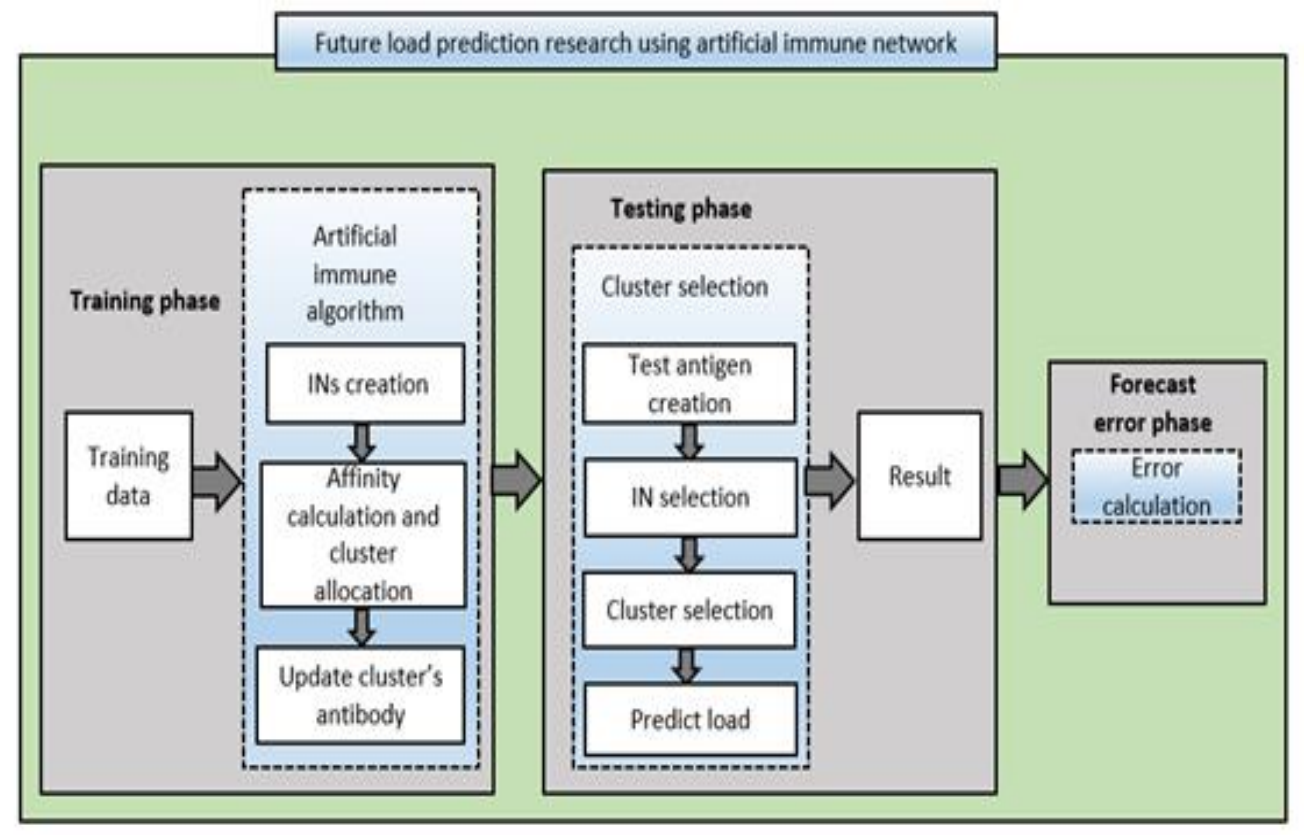

Fig. 1. Structure of proposed approach

Training stage is based on AIN creation, cluster classification, and cluster's antibody upgradation rules for each antigen. Testing stage is based on antigen generation for the predicted date, AIN, and cluster of AIN selection for this antigen and forecasts the antibody of selected cluster as a system load. Error stage has evaluated the performance of proposed approach. An error is calculated between the predicted system load and actual system load.

Notations and terminologies used in this approach are as follows:

Antigen (Ag): An Ag is each hour system load of a day. Immune Networks (INs): An IN is created based on all same hour system load of each day of training data. Ins $=\mathrm{IN}_{1}, \mathrm{IN}_{2}$, ...., $\mathrm{IN}_{24}$, where $\mathrm{IN}_{1}$ is $1^{\text {st }}$ immune network, has 1 st hour system load of each day in training set.

Clusters (C): Each IN has clusters $\mathrm{C}=\mathrm{C}_{1}, \mathrm{C}_{2}, \ldots ., \mathrm{C}_{v}$, where $\mathrm{v}=1,2, \ldots, \mathrm{v}$ indicates the cluster number and $\mathrm{C}_{1}$, $\mathrm{C}_{2}, \ldots, \mathrm{C}_{v}$ each cluster has antigens whose affinity value is $<=\theta$. Affinity value is the difference between antibody of a cluster and the antigen. Antibody (Ab): $\mathrm{An} \mathrm{Ab}$ is the dynamic mean value of all antigens in a cluster. The entire data set is divided into two different day types: holiday and weekday. These two day types are trained and tested separately by our method. 
Algorithm description: Proposed STLF strategy of the immune memory creation can be divided into two-stage training and testing.

\section{A. Training Stage}

Training stage can be summarized in the following steps:

Step 1: Load training dataset

Step 2: Create Immune Networks (INs)

Step 3: For each network

3.1 For each antigen

3.1.1 Affinity calculation and cluster selection

3.1.2 Cluster antibody hypermutation

A flow diagram of the training stage is shown in Figure 2.

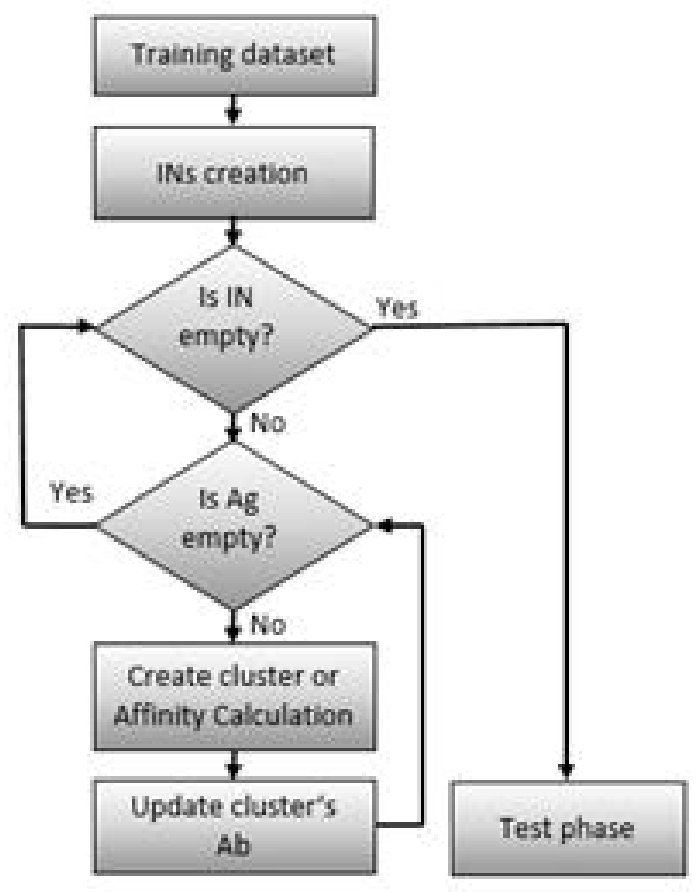

Fig. 2. Flowchart for training stage of IN algorithm

Step 1: Historical dataset is divided into two subsets: training dataset and testing dataset. The first 3/4th sequences of the time series are included in the training set and remaining dataset is included in testing dataset.

Step 2: 24 immune networks are created and components are the following hourly system loads. These system loads are antigens for each immune network.

Step 3: The algorithm stops if all immune networks are trained.
3.1 Until all antigens of an immune network are trained. 3.1.1 Initially if there is no cluster for the immune network, the algorithm creates a new cluster for first antigen and the antigen is set to the cluster's antibody.

For the other antigens of immune network, an affinity is measured between cluster's antibody and the antigen.

$$
\left.\sqrt{\left\{C_{i} A b-C_{i} A g_{j}\right.}\right\}^{2}
$$

Where $\mathrm{d}$ is affinity, $\mathrm{i}=1,2, \ldots ., \mathrm{v}$ indicates cluster's number and $\mathrm{j}$ indicates antigen. If the affinity of the antigen is smaller than or equal to cross-reactivity threshold $(\theta)$, its mean that the antigen lies in this cluster. Affinity for an antigen is calculated by equation (1).

The primary objective of the cluster antibody hypermutation is to improve the diversity of the immune system in order to effectively recognize new antibody of the cluster. The cluster's antibody hypermutation $(\mathrm{H})$ is realized by equation (2).

$$
H=C_{i} A b+C_{i} A g_{j} / 2
$$

The flow diagram and algorithm description of training stage mentioned above are to produce antibodies as the classification rules. In proposed method, each piece of data is defined as an antigen to invade the AIN. If calculated affinity between an antigen and antibody of a cluster is less than the cross-reactivity threshold value $\theta$, the antigen is inserted into the same cluster and the antibody of cluster is updated, otherwise, a new cluster is created and the antigen is set as antibody of the created cluster.

\section{B. Testing Stage}

Testing stage of proposed approach predicts electricity load forecast value of next day by considering an antigen that is created by the mean value of last three days of same hour electricity load forecasted value as a testing antigen. Testing stage of this approach can be summarized as below:

Step 1: Immune network and antigen selection: IN is selected based on the forecast hour of next day and an antigen is created by mean of last three days' actual system loads.

Step 2: Affinity calculation: Affinity is calculated between antibody of each cluster in the selected immune network and the created antigen.

Step 3: Cluster selection: In the identified immune network, a cluster is selected based on the minimum affinity value for the selected antigen. Minimum affinity (MinAf) is calculated based on equation (3). 


$$
\operatorname{MinAf}=\min _{i=1}^{v} A f(i)
$$

Where $A f(i)=\left|C_{i} A b-C_{i} A g\right| a$ nd $\mathrm{i}=1,2, \ldots \ldots, \mathrm{v}$ indicates number of clusters in the selected immune network. Step 4: Load forecast

Antibody of selected cluster is forecasted as system load value for that hour. A flow diagram of the testing stage is shown in Figure 3.

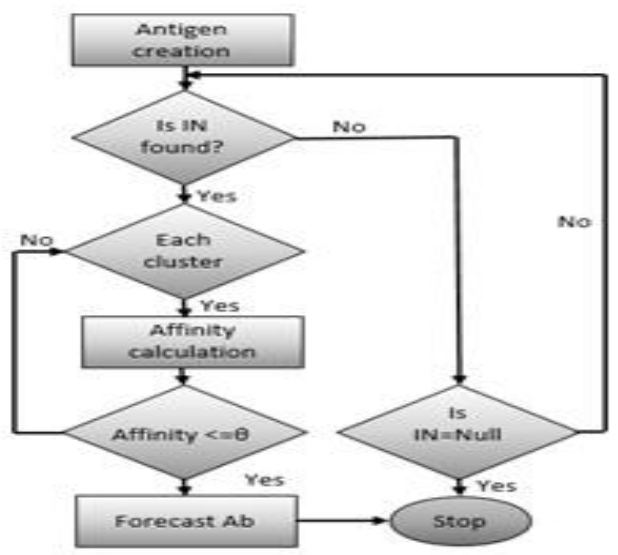

Fig. 3. Flowchart for testing stage of IN algorithm

Error Calculation: Error calculation statistics play a critical role in tracking forecast accuracy, monitoring for exceptions, and benchmarking the forecasting process. We have evaluated post-sample forecasting performance of this method using MAPE, Mean Absolute Error (MAE) calculation. MAPE is calculated by the following equation (4):

$$
M A P E=\frac{1}{24} \sum_{2} 4^{i}=1
$$

\section{EXPERIMENTAL RESULTS}

We have implemented proposed approach of shortterm load forecasting in MATLAB R2014a, on the desktop computer with Intel Core2 Duo E7500@2.94 GHz, 64-bit Windows 10 operating system with 4GB memory (RAM), and the cross- reactivity threshold value $(\theta)$ is 0.1 . Crossreactivity threshold, $\theta \epsilon(0,1)$ is used to decide whether an antigen is joined in a cluster. Only one parameter, cross- reactivity threshold $(\theta)$ is used in this model. The dataset is taken from the website (http://gdudek.el.pcz.pl/varia/stlf- data) for our experiment and dataset description is discussed in Table I.

TABLE 1

DATA SET FOR EXPERIMENT

\begin{tabular}{ll}
\hline \hline Data Symbol & Data Description \\
\hline A & Time series of the hourly loads of the \\
& Polish power system from the period \\
& $2002-2004$
\end{tabular}

Our proposed method takes data from Table I, calculates forecast loads and forecast errors. Actual load, load forecast, and forecast error for the Polish power system in Poland on June 22, 2004 (Tuesday) and December 10, 2004 (Friday) are shown in Table II and April 18, 2004 (Sunday) is shown in Table III. Corresponding actual and predict loads are plotted in Figure 4, Figure 5, and Figure 6 respectively. Besides, MAPE, MAE, Mean Error (ME), Mean Percentage Error (MPE), maximum error, minimum error, maximum absolute error, and minimum absolute error for these test days are shown in Table IV. Low values of MAPE and MAE in Table IV represent high forecast accuracy of our method, which is discussed in Figure 4, Figure 5, and Figure 6. Low values of ME and MPE in Table IV indicate that proposed method has no considerable error bias.

Values of four other indicators of Table IV (low value of MAE) represent stability of predictions of our load forecast method. From Figure 4, Figure 5, and Figure 6, we can also observe that the forecasted load curve accurately follows actual load curve without any sharp deviation. After forecasting of each day, this data window proceeds by 24 hours and load forecast of next day is obtained.

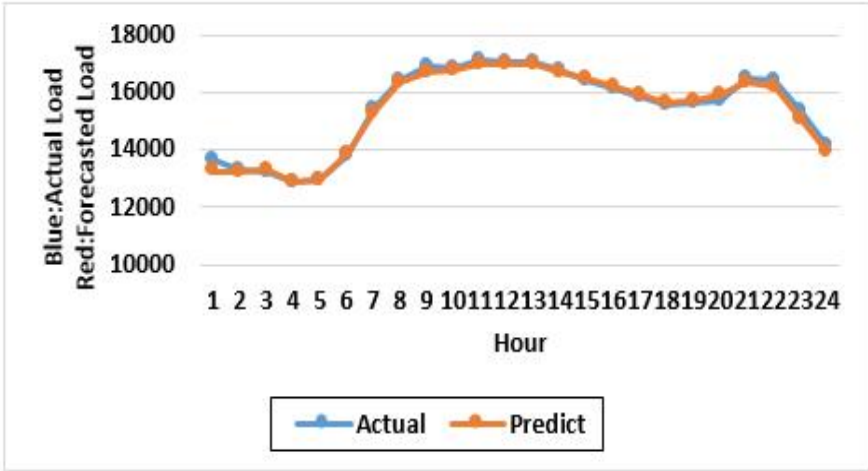

Fig. 4 . Actual load (Blue), forecast load (Red) for Tuesday, June 22, 2004 of Poland 
TABLE 2

ACTUAL LOAD, LOAD FORECAST, FORECAST ERROR AND MAPE OF THE POLISH POWER SYSTEM IN POLAND

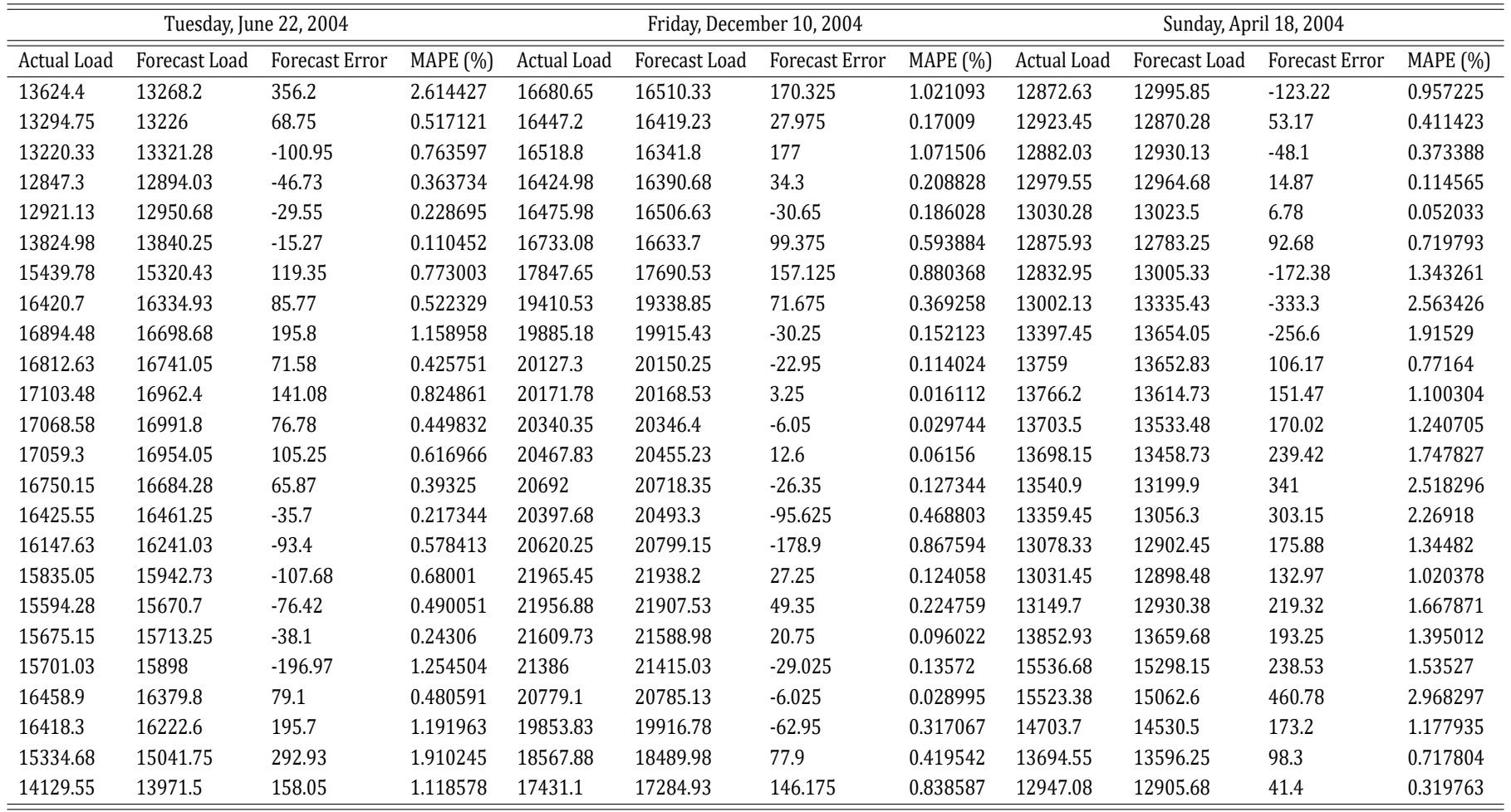

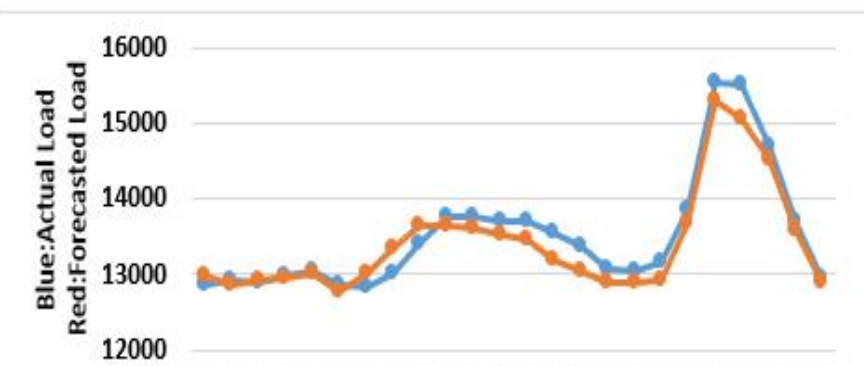

123456789101112131415161718192021222324 Hour

\section{$\longrightarrow$ Actual $\longrightarrow$ Predict}

Fig. 5 . Actual load (Blue), forecast load (Red) for Friday, December 10, 2004 of Poland

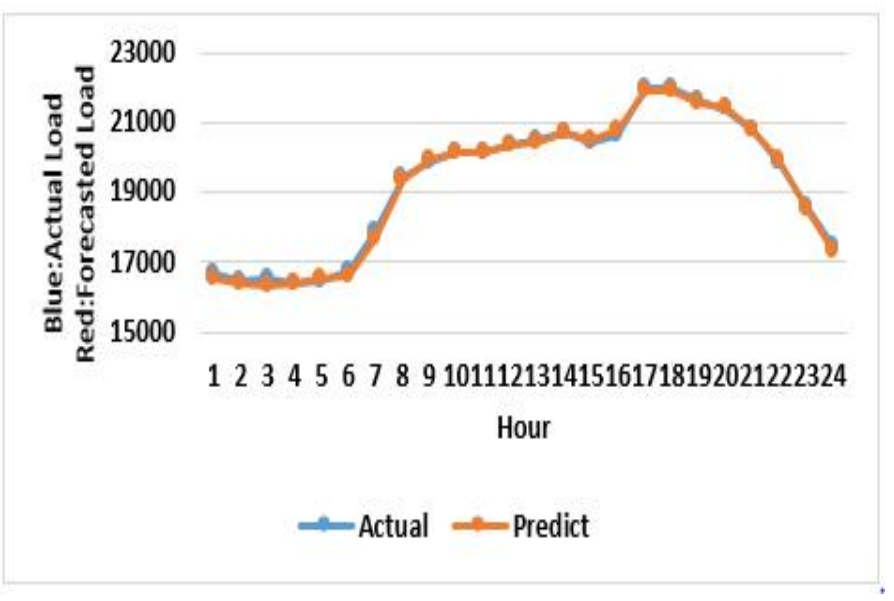

Fig. 6 . Actual load (Blue), forecast load (Red) for Sunday, April 18, 2004 of Poland

TABLE 3

MAPE, MAE, ME, MPE, MAXIMUM ERROR, MINIMUM ERROR, MAXIMUM ABSOLUTE ERROR AND MINIMUM ABSOLUTE ERROR OF THE PROPOSED METHOD OF POLAND DATASET)

\begin{tabular}{|c|c|c|c|c|c|c|c|c|}
\hline & MAPE (\%) & MAE & ME & MPE (\%) & Maximum Error & Minimum Error & Maximum Absolute Error & Minimum Absolute Error \\
\hline Tuesday, June 22, 2004 & 0.746989 & 114.7075 & 52.97667 & 0.336167 & 356.2 & -196 & 356.2 & 15.27 \\
\hline Friday, December 10, 2004 & 0.35513 & 65.15938 & 24.42812 & 0.152843 & 177 & -178.9 & 178.9 & 3.25 \\
\hline Sunday, April 18, 2004 & 1.260229 & 172.7483 & 94.94833 & 0.66418 & 460.78 & -333.3 & 460.78 & 6.78 \\
\hline
\end{tabular}


TABLE 4

MAPE VALUES OF LOAD FORECAST METHOD OF [4], [5] AND OUR PROPOSED METHOD FOR WEDNESDAY (APRIL 21, 2004), POLAND (FOR THE TEST DATASET)

\begin{tabular}{ll}
\hline \hline Methods & MAPE (\%) \\
MLP [4] & 2.02 \\
NN [4] & 1.94 \\
FE [4] & 1.76 \\
AIS [5] & 1.88 \\
Proposed method & 0.94 \\
\hline \hline
\end{tabular}

Our method is compared with the load forecast technique of NN, MLP, FE of [4], and AIS [5]. The MAPE values of load forecast method of [4], [5] and our proposed method are shown in Table $\mathrm{V}$ and observed that the load forecast error of our method in all test periods are considerably lower than load forecast error of methods proposed in [4] and [5]. This comparison clearly shows superiority of our proposed method in comparison to load forecast methods mentioned above.

\section{DISCUSSION}

STLF problem is solved by AIN-based clustering approach. The method has training and testing phase. Training phase is used to train the method and testing phase to test the method. Training phase generates INs and creates clusters for each IN. In testing phase, an antigen is generated and cluster is selected which then forecasts the corresponding antibody of selected cluster, and is treated as system load. A Cross-reactivity threshold value $(\theta)$ is used to tune our method for selecting cluster. The method is validated and tested by the data set taken from the website (http://gdudek.el.pcz.pl/varia/stlf-data) and observed to provide representative prediction of power demand in STLF. The method provides an estimate with greater granularity and better accounting for the variability in system load through the day. This can be of particular use if the climatic conditions are used in STLF to predict system loads in electricity market. Table $\mathrm{V}$ provides a comparison among the results obtained from our method and some popular short-term load forecasting methods. Results from our method presented in Table $\mathrm{V}$ provide more representation than other methods.

\section{CONCLUSION}

Immune system has many interesting features such as classification, clustering, and regression. These features can be used in several prediction and optimization methods. In proposed promising technique, a new prediction formulation of AIN for future load forecast is discussed here, which is state-of-the-art and time to market for short-term load forecasting. The historical data are trained by our AIS learning method, which also forecasts the appropriate matching load for future data from the training set. A cluster is represented by an antibody. The method has evaluated a new antigen, which is mean of last three days' same hour actual load. This antigen is used in testing phase to identify suitable antibody from a cluster and forecast system load for the same hour. Ab and Ag play a major role in the whole time series for future prediction of electricity load. Performance and accuracy of this method are calculated which is better than existing methods.

Climatic conditions are most important in this research because electricity load will be influenced by weather. Here meteorological conditions are not deployed. Further work will be concentrated on establishment of the superior antibody receptor structure and considering of different atmospheric conditions like temperature, humidity, and wind speed for reducing forecast errors.

\section{REFERENCES}

[1] D. Bunn and E. Farmer, "Economic and operational context of electric load prediction," in Comparative Models for Electrical Load Forecasting. New York, NY: John Wiley and Sons Inc., 1985, pp. 3-11.

[2] R. F. Engle, C. Mustafa and J. Rice, "Modelling peak electricity demand," Journal of Forecasting, vol. 11, no. 3, pp. 241-251, 1992. DOI: 10.1002/for.3980110306

[3] G. Gross and F. D. Galiana, "Short-term load forecasting," in IEEE International Congress on Big Data, Mexico, MX, 1987, pp. 17-24. DOI: 10.1109/PROC.1987.13927

[4] G. Dudek, "Neuro-fuzzy approach to the next day load curve forecasting," Przeglad Elektrotechniczny, vol. 87, no. 2, pp. 61-64. 2011.

[5] G. Dudek, "Artificial immune system for short-term electric load forecasting," in 9th International Conference Artificial Intelligence and Soft Computing, Berlin, Germany, 2008, pp. 1007-1017, 2008.

DOI: $10.1007 / 978-3-540-69731-2 \_95$

[6] G. T. Heinemann, D. A. Nordmian and E. Plant, "The relationship between summer weather and summer loadsa regression analysis," IEEE Transactions on Power Apparatus and Systems, vol. 85, no. 11, pp. 1144-1154, 1966. DOI: $10.1109 /$ TPAS.1966.291535 
[7] H. Hahn, S. Meyer-Nieberg and S. Pickl, "Electric load forecasting methods: Tools for decision making," European Journal of Operational Research, vol. 199, no. 3, pp. 902-907, 2009. DOI: 10.1016/j.ejor.2009.01.062

[8] E. H. Barakat, M. A. Qayyum, M. N. Hamed and S. A. Al Rashed, "Short-term peak demand forecasting in fast developing utility with inherit dynamic load characteristics. I. Application of classical time-series methods. II. Improved modelling of system dynamic load characteristics," IEEE Transactions on Power Systems, vol. 5, no. 3, pp. 813-824, 1990. DOI: 10.1109/59.65910

[9] S. J. Huang and K. R. Shih, "Short-term load forecasting via ARMA model identification including non-Gaussian process considerations," IEEE Transactions on Power Systems, vol. 18, no. 2, pp. 673-679, 2003. DOI: 10.1109/TPWRS.2003.811010

[10] G. Juberias, R. Yunta, J. G. Moreno and C. Mendivil, "A new ARIMA model for hourly load forecasting,' in IEEE Conference on Transmission and Distribution, New Orleans, LA, 1999. DOI: 10.1109/TDC.1999.755371

[11] K. Liu, S. Subbarayan, R. R. Shoults, M. T. Manry, C. Kwan, F. I. Lewis and J. Naccarino, "Comparison of very shortterm load forecasting techniques," IEEE Transactions on Power Systems, vol. 11, no. 2, pp. 877-882, 1996. DOI: 10.1109/59.496169

[12] S. J. Kiartzis and A. G. Bakirtzis, "A fuzzy expert system for peak load forecasting: Application to the Greek power system," in 10th Mediterranean Electrotechnical Conference-MELECON, Lemesos, Cyprus, 2000.

[13] V. Miranda and C. Monteiro, "Fuzzy inference in spatial load forecasting," in IEEE Power Engineering Winter Meeting, Singapore, 2000, pp. 1063-1068. DOI: 10.1109/PESW.2000.850087

[14] S. E. Skarman and M. Georgiopoulous, "Short-term electrical load forecasting using a fuzzy ARTMAP neural network," in Applications and Science of Computational Intelligence, 1998, pp. 181-191.

[15] D. E. Rumelhart and J. L. McClelland, Parallel Distributed Processing: Explorations in the Microstructure of Cogniton. Cambridge, MA: MIT Press, 1986.

[16] A. G. Bakirtzis, V. Petridis, S. J. Kiartzis, M. C. Alexiadis and A. H. Maissis, "A neural network short-term load forecasting model for the Greek power system," IEEE Transactions on Power Systems, vol. 11, no. 2, pp. 858-863, 1996. DOI: 10.1109/59.496166

[17] H. Mori and N. Kosemura, "Optimal regression tree based rule discovery for short-term load forecasting," in IEEE Power Engineering Society Transmission and Distribution Conference, Vancouver, Canada, 2001, pp. 421-426. DOI: 10.1109/PESW.2001.916878
[18] T. W. S. Chow and C. T. Leung, "Nonlinear autoregressive integrated neural network model for short-term load forecasting," in IEEE Conference on Generation, Transmission and Distribution, Stevenage, UK, 1996, pp. 500-506. DOI: 10.1049/ip-gtd:19960600

[19] D. Dasgupta, "Advances in artificial immune systems," IEEE Computational Intelligence Magazine, vol. 1, no. 4, pp. 40-49, 2006. DOI: 10.1109/MCI.2006.329705

[20] M. El-Abd, "Performance assessment of foraging algorithms vs. evolutionary algorithms," Information Sciences, vol. 182, no. 1, pp. 243-263, 2012. DOI: 10.1016/j.ins.2011.09.005

[21] L. N. De Castro and J. I. Timmis, "Artificial immune systems as a novel soft computing paradigm," Soft Computing, vol. 7, no. 8, pp. 526-544, 2003. DOI: 10.1007/s00500-002-0237-z

[22] I. Roitt and J. Brostoff, Immunology 5E. New York, NY: Mosby Int. Ltd., 1998.

[23] S. N. Qasem, S. M. Shamsuddin and A. M. Zain, "Multiobjective hybrid evolutionary algorithms for radial basis function neural network design," KnowledgeBased Systems, vol. 27, pp. 475-497, 2012. DOI: 10.1016/j.knosys.2011.10.001

[24] Z. Yudong and W. Lenan, "Stock market prediction of SandP 500 via combination of improved BCO approach and BP neural network," Expert Systems with Applications, vol. 36, no. 5, pp. 8849-8854, 2009. DOI: 10.1016/j.eswa.2008.11.028

[25] E. Hadavandi, H. Shavandi and A. Ghanbari, "Integration of genetic fuzzy systems and artificial neural networks for stock price forecasting," Knowledge-Based Systems, vol. 23, no. 8, pp. 800-808, 2010. DOI: 10.1016/j.knosys.2010.05.004

[26] L. N. De Castro and F. J. Von Zuben, "Learning and optimization using the clonal selection principle," IEEE Transactions on Evolutionary Computation, vol. 6, no. 3, pp. 239-251, 2002 . DOI: 10.1109/TEVC.2002.1011539

[27] L. N. De Castro and F. J. Von Zuben, "The clonal selection algorithm with engineering applications," in The Genetic and Evolutionary Computation Conference (GECCO), Las Vegas, NV, 2000, pp. 36-39.

[28] A. Watkins, J. Timmis and L. Boggess, "Artificial Immune Recognition System (AIRS): An immune-inspired supervised learning algorithm," Genetic Programming and Evolvable Machines, vol. 5. No. 3, pp. 291-317, 2004. DOI: $10.1023 / B: G E N P .0000030197 .83685 .94$

[29] A. Secker and A. Freitas, "Wairs: Improving classification accuracy by weighting attributes in the airs 
classifier," in IEEE Congress on Evolutionary Computation, Singapore, pp. 3759-3765, 2007. DOI: 10.1109/CEC.2007.4424960

[30] N. K. Jerne, "Towards a network theory of the immune system," Annales D'Immunologie, 125, no 1-2, pp. 373-389, 1974.

[31] J. Timmis and M. Neal, "A resource limited artificial immune system for data analysis," Knowledge-Based Systems, vol. 14, no. 3, pp. 121-130, 2000.

[32] A. M Acilar and A. Arslan, "A collaborative filtering method based on artificial immune network," Expert Systems with Applcations, vol. 36, no. 4, pp. 8324-8332, 2009. DOI: $10.1016 /$ j.eswa.2008.10.029
[33] S. Cayzer and U. Aickelin, "A recommender system based on idiotypic artificial immune networks," Journal of Mathematical Model and Algorithms, vol. 4, no. 2, pp. 181-198, 2005. DOI: 10.1007/s10852-004-5336-7

[34] M. H. Chen, C. H. Teng and P. C. Chang, "Applying artificial immune systems to collaborative filtering for movie recommendation," Advanced Engineering Informatics, vol. 29, no. 4, pp. 830-839, 2014. DOI: 10.1016/j.aei.2015.04.005

[35] C. Tsao, P. Chang and C. Fan, "A patent quality classification model based on an artificial immune system," Soft Computing, vol. 20, no. 187, pp. 1-10. 2016.

— This article does not have any appendix. - 